\title{
Evaluation of newborn screening for medium chain acyl-CoA dehydrogenase deficiency in 275000 babies
}

\author{
K Carpenter, V Wiley, K G Sim, D Heath, B Wilcken
}

\begin{abstract}
Objective-To evaluate newborn screening by tandem mass spectrometry for detection of medium chain acyl-CoA dehydrogenase (MCAD) deficiency, a fatty acid oxidation disorder with significant mortality in undiagnosed patients.

Design-The following were studied: $(a)$ 13 clinically detected MCAD deficient subjects, most homozygous for the common A985G mutation, whose newborn screening sample was available; (b) 275653 consecutive neonates undergoing routine newborn screening. Screened infants with blood octanoylcarnitine levels $\geqslant 1 \mu \mathrm{mol} / 1$ were analysed for the A985G mutation, had analysis of plasma and repeat blood spot acylcarnitines and urinary organic acids, and had fibroblast fatty acid oxidation or acylcarnitine studies.

Result-Twelve of the 13 patients later diagnosed clinically had newborn octanoylcarnitine levels $>2.3 \mu \mathrm{mol} / 1$. Twenty three screened babies had initial octanoylcarnitine levels $\geqslant 1 \mu \mathrm{mol} / 1$. Eleven of 12 babies with persistent abnormalities had metabolite and/or enzyme studies indicating MCAD deficiency. Only four were homozygous for the A985G mutation, the remainder carrying one copy.

Conclusions-Most patients with symptomatic MCAD deficiency could be detected by newborn screening. Infants actually detected had a lower frequency of A985G alleles than clinically diagnosed cases and may have a lower risk of becoming symptomatic.
\end{abstract}

(Arch Dis Child Fetal Neonatal Ed 2001;85:F105-F109)

New South Wales Newborn Screening Programme, The Children's Hospital at Westmead, Sydney, Australia

V Wiley

D Heath

B Wilcken

Biochemical Genetics Service, The Children's Hospital at Westmead

K Carpenter K G Sim

B Wilcken

Correspondence to: Dr Wilcken, The Children's Hospital at Westmead, Locked Bag 4001

Westmead, NSW 2145,

Australia

Bridgetw@chw.edu.au
Keywords: acylcarnitines; octanoylcarnitine; fatty acid oxidation; acylglycines; Reye syndrome

Fatty acid oxidation in the mitochondria is an essential source of cellular energy. Medium chain acyl-CoA dehydrogenase (MCAD) deficiency is the commonest disorder of mitochondrial fatty acid oxidation. It is recessively inherited, and is particularly common in populations of northern European origin. ${ }^{1}$ Several studies have shown that about $80 \%$ of clinically ascertained cases are homozygous for an A to $G$ transition at position 985, resulting in a lysine to glutamate substitution, ${ }^{2-4}$ with almost all of the remainder having one copy of this mutation together with some other rare mutation.

MCAD deficiency may result in episodic hypoketotic hypoglycaemia and hepatic encephalopathy triggered by periods of catabolic stress, suggestive of Reye syndrome. Episodes occur most often between 3 months and 6 years of age, but a proportion of the diagnosed patients have symptoms in the neonatal period, sometimes with a fatal outcome. ${ }^{5}$ An overall mortality of about $25 \%$ has been reported, ${ }^{6-8}$ but it is clear that some people remain asymptomatic throughout life. ${ }^{9}$ Almost all deaths have been in previously undiagnosed patients. After diagnosis, institution of management to avoid fasting stress, especially during intercurrent illnesses, appears effective in avoiding serious morbidity and mortality. ${ }^{6}$ Patients with MCAD deficiency have raised urinary acylglycines, even when well, and have impaired oxidation of medium chain fatty acids, which can be detected in cultured skin fibroblasts. ${ }^{10}$

Mass screening for MCAD deficiency (and other disorders) has become practicable through the use of tandem mass spectrometry to analyse acylcarnitines in dried blood spots. ${ }^{11}$ In the United Kingdom, two health technology assessments have examined the implications of expanded newborn screening, including screening for MCAD deficiency. ${ }^{12} 13$ The second study suggested that among many disorders potentially detectable in neonates by tandem mass spectrometry, a case could only be made for introducing screening for MCAD deficiency and glutaric aciduria type 1, and recommended monitoring of the clinical effectiveness and cost, although such an evaluation has not yet taken place. ${ }^{14}$ The recommendations for MCAD deficiency were based in part on the clear benefit from early detection and management and also on a calculated incidence of MCAD deficiency derived from A985G carrier frequency. However, early results from such programmes have indicated a much lower frequency of homozygotes for the A985G mutation than expected, and a higher frequency of compound heterozygotes..$^{15} 16$

The NSW Newborn Screening Programme screens all babies born in New South Wales and the Australian Capital Territory (about 95000 births per year), and has used tandem mass spectrometry since April 1998. Parents may dissent from the screening tests as a whole, are handed a descriptive brochure, and are informed by nursing staff about what tests are to be carried out. Randomised controlled trials of screening for such individually rare disorders are not practicable because of the enormous numbers that would be needed for adequate power. ${ }^{17}$ Other levels of evidence must be taken into account. In this study, we report the results of screening for MCAD deficiency in over a quarter of a million neonates. 
Methods

SUBJECTS

Newborn screening samples were collected from $>99 \%$ of babies born in New South Wales and the Australian Capital Territory between April 1998 and March 2001.The total number of babies tested was 275 653. Median age at sampling was 3 days, and over $99 \%$ of babies were sampled before day 6. Samples collected before 48 hours of life because of early discharge $(1.9 \%)$ were deemed inappropriate, and second samples were collected from those babies. However, the initial samples were assayed. Stored newborn screening samples were also available from 13 babies born between January 1981 and June 1997 who were subsequently diagnosed as suffering from MCAD deficiency. Twelve of the samples were taken on days 4-6, with one taken on day 10 . This comprised all the available newborn samples from babies with MCAD deficiency.

LABORATORY METHODS

Acylcarnitines were analysed as butyl esters on a Micromass Quattro II tandem mass spectrometer as previously described. ${ }^{18}$ Mutational analysis for A985G was based on the method of Gregersen. ${ }^{19}$ Urinary organic acids were extracted with ethyl acetate and analysed as their trimethylsilyl esters on a Hewlett-Packard $5890 \mathrm{GC}$ and $5971 \mathrm{MSD}$. Assays of the rate of fatty acid oxidation in cultured skin fibroblasts monitored tritium release with oleate and myristate as substrates. ${ }^{20}$

Fibroblast acylcarnitine accumulation in culture medium was analysed by tandem mass spectrometry after incubation with palmitate and carnitine for 72 hours. Results are expressed as means of replicate (between two and six) analyses. ${ }^{21}$ In two patients, a specific electron transfer flavoprotein (ETF) based assay for MCAD activity was performed by Dr C Vianey-Saban (Hôpital Debrousse, Lyons, France).

NEWBORN SCREENING PROTOCOL

All samples with octanoylcarnitine $\geqslant 0.8$ $\mu \mathrm{mol} / 1$ were assayed again in duplicate in the laboratory. If the octanoylcarnitine level in the final assay was $\geqslant 1 \mu \mathrm{mol} / 1$, a second dried blood sample together with a plasma and urine sample were requested, and analysis for the common A985G mutation performed. At clinical follow up, a skin biopsy was performed for culture of fibroblasts.

\section{CRITERIA FOR ASSIGNMENT OF DIAGNOSIS OF}

MCAD

Patients were diagnosed with MCAD deficiency if one or more of the following criteria were met: homozygous for A985G mutation; raised hexanoylglycine and suberylglycine in urine, increased hexanoylcarnitine, octanoylcarnitine, or decenoylcarnitine in plasma; studies of fibroblast fatty acid oxidation rate with reduced myristate oxidation and an oleate to myristate oxidation ratio $>1.3$; or accumulation of medium chain acylcarnitines in culture medium from skin fibroblasts, with octanoyl carnitine $>1.1 \mathrm{nmol} / \mathrm{mg}$ protein/72 hours. In two cases in which ETF assays were performed, the results were taken as the definitive measure and the diagnosis of affected or carrier assigned.

\section{Results}

RETROSPECTIVE ANALYSIS OF NEWBORN SCREENING SAMPLES FROM PATIENTS LATER DIAGNOSED WITH MCAD DEFICIENCY, AND DEVELOPMENT OF A CUT OFF VALUE

We performed retrospective analysis of newborn screening cards from 13 patients diagnosed clinically with MCAD deficiency. Eleven of the 13 were homozygous for the common MCAD mutation A985G, and two were heterozygous. Twelve had octanoylcarnitine concentrations well in excess of $1 \mu \mathrm{mol} / 1$ (range 2.4-6.8). The remaining baby, ${ }^{22}$ who was homozygous for the common mutation, developed hypoglycaemia and hepatic encephalopathy on day 2 , leading to carnitine depletion, and had an octanoylcarnitine level of 0.27 $\mu \mathrm{mol} / 1$. Prospective analysis of newborn screening samples from 24000 newborns showed $99.2 \%$ to have octanoylcarnitine concentrations of $<0.3 \mu \mathrm{mol} / 1$, all the remainder falling between 0.3 and $0.7 \mu \mathrm{mol} / 1$. None had a level greater than $1 \mu \mathrm{mol} / 1$, and this level was chosen as the cut off value for prospective screening. ${ }^{18}$ The level of octanoylcarnitine did not alter appreciably in newborn screening samples stored for up to three years, and in no case did it increase (data not shown).

PROSPECTIVE NEWBORN SCREENING RESULTS Distribution of octanoylcarnitine concentrations in dried blood spots did not vary greatly with birth weight or day of sampling.

Samples from 23 babies had octanoylcarnitine values greater than $1 \mu \mathrm{mol} / 1$ in the initial dried blood spot sample. All were tested for the common A985G mutation, and a repeat dried blood spot sample requested. Eleven of the 23 did not carry A985G on either allele, and in six of these the octanoylcarnitine had normalised on repeat sampling. One baby had a blood octanoylcarnitine concentration of $1.5 \mu \mathrm{mol} / 1$ on retesting, but further investigation of plasma carnitines and urinary acylglycines did not indicate MCAD deficiency (data not shown). The remaining four infants died from a variety of causes before a second sample could be collected. One had hydrops foetalis. MCAD deficiency was eliminated on enzyme analysis in cultured skin fibroblasts. Three others died before further investigations were possible, but information obtained from the responsible clinicians, including postmortem findings which did not show any increased hepatic fat, did not suggest that MCAD deficiency could have been the cause of death.

Twelve infants had persistently abnormal acylcarnitines and carried at least one copy of A985G. These were investigated further by analysis of urinary organic acids and/or quantitation of plasma carnitine and acylcarnitine. Six patients had a skin biopsy, and studies of oxidation rate were performed on cultured skin fibroblasts. Table 1 summarises the results. Overall 11 patients had results that were clearly 
Table 1 Initial dried blood spot and follow up assays in 12 babies with persistently increased octanoylcarnitine and at least one copy of A985G

\begin{tabular}{|c|c|c|c|c|c|c|c|c|c|c|c|c|}
\hline \multirow[b]{3}{*}{ Patient } & \multicolumn{2}{|c|}{ Newborn screening } & \multicolumn{5}{|c|}{ Biochemical genetics follow up } & \multicolumn{5}{|l|}{ Fibroblast results } \\
\hline & \multirow{2}{*}{$\begin{array}{l}\text { Octanoyl- } \\
\text { carnitine } \\
\text { ( } \text { umol/l) } \\
\text { (cut off }<1 \text { ) }\end{array}$} & \multirow{2}{*}{$\begin{array}{l}\text { A985G } \\
\text { mutational } \\
\text { analysis }\end{array}$} & \multicolumn{2}{|c|}{ Urinary acylglycines } & \multicolumn{3}{|c|}{$\begin{array}{l}\text { Plasma carnitines } \\
(\mu \mathrm{mol} / \mathrm{l})\end{array}$} & \multirow{2}{*}{$\begin{array}{l}\text { Fatty acid oxidation } \\
\text { rate assay \% of } \\
\text { intrabatch controls } \\
O: M \text { ratio }(0.7-1.3)\end{array}$} & \multicolumn{3}{|c|}{ Acylcarnitine profile (nmol/mg/72 h) } & \multirow{2}{*}{$\begin{array}{l}\text { ETF assays } \\
\text { MCAD } \\
\text { activity (Dr } \\
\text { Vianey-Saban) } \\
(2.42(0.61) \\
n=50)\end{array}$} \\
\hline & & & Hexanoyl & Suberyl & $\begin{array}{l}C 6 \\
(<0.1)\end{array}$ & $\begin{array}{l}C 8 \\
(<0.3)\end{array}$ & $\begin{array}{l}\text { C10:1 } \\
(<0.3)\end{array}$ & & $\begin{array}{l}\text { C6 } \\
(0.2-1.4)\end{array}$ & $\begin{array}{l}C 8 \\
(0.1-1.1)\end{array}$ & $\begin{array}{l}C 10 \\
(0.6-1.6)\end{array}$ & \\
\hline 1 & 24.6 & $+/-$ & +++ & +++ & 0.6 & 2.0 & 0.3 & NP & 0.81 & 2.06 & 0.34 & $\mathrm{NP}$ \\
\hline 2 & 14.2 & $+/+$ & $\mathrm{NP}$ & $\mathrm{NP}$ & 1.3 & 5.6 & 0.5 & NP & NP & NP & NP & NP \\
\hline 3 & 13.0 & $+/-$ & +++ & +++ & 1.0 & 3.8 & 0.6 & $\begin{array}{l}\text { Ole } 63 \% \text {, Myr } 10 \% \\
\text { O:M } 6.4\end{array}$ & 2.90 & 6.97 & 1.25 & NP \\
\hline 4 & 8.6 & $+/-$ & +++ & +++ & 0.9 & 3.8 & 0.3 & NP & 1.56 & 4.07 & 0.66 & $\mathrm{NP}$ \\
\hline 5 & 8.0 & $+/+$ & +++ & +++ & 0.5 & 2.6 & 0.3 & $\mathrm{NP}$ & $\mathrm{NP}$ & & & $\mathrm{NP}$ \\
\hline 6 & 8.0 & $+/+$ & +++ & +++ & 1.3 & 8.4 & 2.5 & NP & NP & & & $\mathrm{NP}$ \\
\hline 7 & 8.0 & $+/+$ & +++ & +++ & 1.1 & 5.7 & 0.7 & $\mathrm{NP}$ & $\mathrm{NP}$ & & & NP \\
\hline 8 & 4.9 & $+/-$ & ++ & ++ & 0.2 & 1.1 & 0.4 & $\begin{array}{l}\text { Ole } 51-87 \% \text {, Myr } \\
31-52 \% \\
\text { O:M } 1.6-1.7\end{array}$ & 1.60 & 3.07 & 1.43 & $\mathrm{NP}$ \\
\hline 9 & 3.5 & $+/-$ & + & ++ & 1.1 & 3.1 & 0.4 & NP & $\mathrm{NP}$ & & & $\mathrm{NP}$ \\
\hline 10 & 3.3 & $+/-$ & + & + & 0.2 & 0.7 & 0.2 & $\begin{array}{l}\text { Ole } 107 \%, \text { Myr } \\
38 \% \\
\text { O:M } 2.8\end{array}$ & 2.35 & 7.81 & 4.01 & NP \\
\hline 11 & 1.1 & $+/-$ & TR, ND & TR, ND & 0.7 & 1.9 & 1.0 & $\begin{array}{l}\text { Ole } 41-59 \%, \text { Myr } \\
42-44 \% \\
\text { O:M } 1.0-1.3\end{array}$ & 0.92 & 1.03 & 0.67 & $0.44,0.31$ \\
\hline 12 & 1.1 & $+/-$ & $\mathrm{ND}$ & ND & $\mathrm{NP}$ & $\mathrm{NP}$ & NP & $\begin{array}{l}\text { Ole } 32 \% \text {, Myr } 22 \% \\
\text { O:M } 1.5\end{array}$ & 1.45 & 1.52 & 1.11 & $1.28,1.21$ \\
\hline
\end{tabular}

Patients 1-11 were assigned a diagnosis of medium chain acyl-CoA dehydrogenase (MCAD) deficiency, and patient 12 was considered to be a carrier at low risk of developing symptoms. +/+, homozygous for A985G; +/-, heterozygous for A985G; Ole, oleate; Myr, myristate; O:M, oleate to myristate ratio; C4, butyrylcarnitine; C6, hexanoylcarnitine; C8, octanoylcarnitine; C10, decanoylcarnitine; C10:1, decenoylcarnitine; ND, not detected; NP, not performed; NI, not increased; +++, grossly elevated; ++, moderately increased; + , slightly increased; TR, trace increase.

consistent with MCAD deficiency. Four were homozygous for the common mutation, ten had clearly increased urinary acylglycines (one had not had urine collected), and in six who had skin biopsies, there was decreased oxidation of myristate and an increased oleate to myristate oxidation ratio in cultured skin fibroblasts or pronounced accumulation of octanoylcarnitine on fibroblast acylcarnitine profiling. One further patient (patient 11) was classified as MCAD deficient on the basis of decreased MCAD activity in cultured skin fibroblasts using a specific ETF based assay performed by Dr C Vianey-Saban. This patient had intermittently raised urinary acylglycines and decreased oxidation of myristate in cultured skin fibroblasts, but the rate of oleate oxidation was also decreased, and the oleate to myristate ratio was not typical of MCAD deficiency (table 1 ).

PREVALENCE OF THE A985G MUTATION IN MCAD DEFICIENT PATIENTS

The 11 babies classified as MCAD deficient in the newborn screening cohort carried the common mutation on 15 of the 22 alleles. There were 17 MCAD deficient patients clinically diagnosed in New South Wales before screening began. Sixteen of these had had DNA analysis for the common mutation, which was carried on 29 of the 32 alleles, a non-significant difference (Fisher's Exact Test).

\section{Discussion}

MCAD deficiency has been considered appropriate for inclusion in newborn screening programmes because it is a disorder with significant morbidity and mortality, and estimates of incidence based on mutation testing suggest the defect remains underdiagnosed. ${ }^{4}$ Several studies have shown that around $80 \%$ of patients presenting clinically are homozygous for the AG985 mutation, with about $18 \%$ being compound heterozygotes. ${ }^{23}$ These figures are in keeping with our own experience in New South Wales, Australia where 29 of 32 alleles from 16 of our clinically presenting patients carried the A985G mutation. Combined figures from three neonatal population studies in Australia (one each in New South Wales, Victoria, and Queensland) have shown the prevalence of A985G carriers to be $1: 86$ (17 of 1461). This implies a birth prevalence of homozygous A985G MCAD deficiency of 1:29 500 (95\% confidence interval $1: 11500$ to $1: 87000$ ) and an overall birth prevalence of MCAD deficiency of $1: 19000 .^{24} 25$

As in most other centres, before introduction of newborn screening, our rate of diagnosis of MCAD deficiency was considerably lower than that predicted by the carrier frequency for A985G and the frequency of this mutation in MCAD alleles of clinically diagnosed patients. From 1980 to 1997 we would have expected 86 cases of MCAD deficiency, but only 15 were clinically ascertained. Seven of these were born between 1990 and 1997, the years immediately before screening began, when 38 would have been expected. The assumption was that the missing patients had had no or very mild symptoms or had died without a diagnosis being made. The introduction of newborn screening has led to the discovery of 11 new patients in 36 months, but only four of these are homozygous for the common mutation. Several groups have reported this unexpected mutational spectrum from patients identified by newborn screening. ${ }^{15}{ }^{16-28}$ Our finding of 4:275 653 (1:68 913) patients homozygous for A985G is considerably lower than the predicted prevalence from Australian figures but is still within the rather wide $95 \%$ confidence interval. 
Two important questions arise from these findings. If the carrier frequencies are correct, and there is considerable agreement from many studies of populations derived from Northern Europe, could there be some homozygous A985G individuals who have normal acylcarnitine concentrations during the neonatal period and are thus missed on newborn screening? Secondly, do all those presumed compound heterozygotes detected by screening have the same risk of death and disability as homozygotes, or are we detecting and treating some patients who have very little chance of becoming symptomatic?

Our retrospective analysis of newborn screening cards from previously diagnosed patients suggests that patients with later symptoms are very unlikely to have had normal acylcarnitine profiles in the newborn period. The one baby who did have a normal octanoylcarnitine concentration was homozygous for the A985G mutation, had severe hepatic encephalopathy as a neonate, and had carnitine depletion. ${ }^{22}$ No other acylcarnitine measure or ratio would have distinguished her from healthy neonates. It remains possible that not all patients homozygous for the A985G mutation will show raised octanoylcarnitine in the neonatal period. Maybe other polymorphisms, perhaps affecting chaperonins, could offer protection by stabilising the mutant protein and normalising enzyme activity, thus accounting for the "missing" cases.

The non-A985G mutations in our compound heterozygotes are probably rare and diverse. The question of whether patients with certain mutations are likely to be mildly affected, with high residual enzyme activities, has been investigated by expression studies, but a correlation of genotype to phenotype is not straightforward.$^{29}$ It is important to remember, however, that these individuals are being detected because of a raised octanoylcarnitine, which in itself is a clear indication of a functional impairment of their ability to metabolise fatty acids. It is very likely that environmental or other genetic factors will determine if an affected individual becomes symptomatic. One of our compound heterozygote neonates (patient 1, table 1) did indeed become hypoglycaemic, requiring transfer to a neonatal intensive care unit, but has remained well since on conservative treatment. She had very high octanoylcarnitine concentration in her newborn screening sample.

Two cases illustrate the problems of atypical follow up results in heterozygous A985G infants detected by screening. Patient 12 had considerably reduced rates of oxidation of both medium and long chain fatty acids in cultured skin fibroblasts, but a specific assay for MCAD activity showed results in the heterozygous MCAD range (table 1). His risk of developing symptoms is unknown, but he may well carry a defect in another part of the fatty acid oxidation pathway that results in his biochemical findings. Patient 11 had urine, plasma, and fibroblast results atypical for MCAD deficiency, but her specific ETF based assay of MCAD activity suggests that she does have
MCAD deficiency. However, mutational analysis of all exons in the gene failed to identify another mutation (Professor B Andreson, Aarhus University, Denmark).

It is clear from our retrospective study that newborn screening using tandem mass spectrometry can detect almost all patients with MCAD deficiency who would later have presented with symptoms, and some of these will certainly be saved from death or morbidity with simple and appropriate management. Some other babies are detected who have abnormal MCAD activity, but may have a benign disorder. At present it is not possible to distinguish these cases from those at risk of decompensation, and all will require counselling and a stringent management plan. Thus, decisions on expansion of newborn screening programmes to include MCAD deficiency should be taken in the knowledge that this is a complex disorder, and that not all cases discovered by newborn screening may be at risk. Screening programmes uncover unexpected findings, and these are not always predictable from theoretical projections. ${ }^{12} 1330$

Professor Brage Andresen, Aarhus, sequenced the exons of the MCAD gene in one of the patients. Dr Christine Vianey-Saban, Lyons, performed an ETF based enzyme assay on skin fibroblasts from two of the patients. All staff of the NSW Newborn Screening Programme and the staff of the NSW Biochemical Genetics Service gave valuable help in different aspects of the study.

1 Tanaka K, Gregersen N, Ribes A, et al. A survey of the newborn populations in Belgium, Germany, Poland, Czech 作 Republic, Hungary, Bulgaria, Spain, Turkey, and Japan for the G985 variant allele with haplotype analysis at the medium chain acyl-CoAdehydrogenase gene locus: clinical and volutionary consideration. Pediatr Res 1997,41:201-9.

Blakemore AI, Singleton H, Pollitt RJ, et al. Frequency of the G985 MCAD mutation in the general population [letter]. Lancet 1991;337:298-9.

3 Curtis D, Blakemore AI, Engel PC, et al. Heterogeneity for mutations in medium chain acyl-CoA dehydrogenase deficiency in the UK population. Clin Genet 1991;40:283-6.

4 Pollitt RJ, Leonard JV. Prospective surveillance study of medium chain acyl-CoA dehydrogenase deficiency in the UK. Arch Dis Child 1998;79:116-19.

5 Wilcken B, Carpenter KH, Hammond J. Neonatal symptoms in medium chain acyl coenzyme A dehydrogenase deficiency. Arch Dis Child 1993;69:292-4.

6 Iafolla AK, Thompson RJJ, Roe CR. Medium-chain acyl-coenzyme A dehydrogenase deficiency: clinical course in 120 affected children. $\mathcal{F}$ Pediatr 1994;124:40915.

7 Touma EH, Charpentier C. Medium chain acyl-CoA dehydrogenase deficiency. [Review] Arch Dis Child 1992;67:142-5.

8 Wilcken B, Hammond J, Silink M. Morbidity and mortality in medium chain acyl coenzyme A dehydrogenase deficiency. Arch Dis Child 1994;70:410-12.

9 Heptinstall LE, Till J, Wraith JE, et al. Common MCAD mutation in a healthy parent of two affected siblings. 7 Inherit Metab Dis 1995;18:638-9.

10 Stanley C. Disorders of fatty acid oxidation. In: Fernandez J, Saudubray JM, van den Berghe G, eds. Inborn metabolic disease. Berlin: Springer-Verlag, 2000;139-50.

11 Naylor EW, Chace DH. Automated tandem mass spectrometry for mass newborn screening for disorders in fatty acid, organic acid, and amino acid metabolism. 7 Child Neurol 1999; 14(suppl 1):S4-8.

12 Pollitt RJ, Green A, McGill J, et al. Neonatal screening for inborn errors of metabolism: cost, yield and outcome. inborn errors of metabolism: cost,

13 Seymour CA, Thomason MJ, Chalmers RA, et al. Newborn screening for inborn errors of metabolism: a systematic review. [Review] Health Technol Assess 1997;1:i-iv.

14 Tanner S, Sharrard M, Cleary M, et al. Screening for medium chain acyl-CoA dehydrogenase deficiency has still not been evaluated. BMF 2001;322:112.

15 Marsden D, Zykovicz T, Larson C, et al. Spectrum of expression of MCAD and SCAD detected by newborn screening. F Inherit Metab Dis 2000;23(suppl 1):14.

16 Ziadeh R, Hoffman EP, Finegold DN, et al. Medium chain acyl-CoA dehydrogenase deficiency in Pennsylvania: neonatal screening shows high incidence and unexpected natal screening shows high incidence and unexp
mutation frequencies. Pediatr Res 1995;37:675-8.

17 Wilcken B. Rare diseases and the assessment of intervention. F Inherit Metab Dis 2001 ; in press. 
18 Wiley V, Carpenter K, Wilcken B. Newborn screening with tandem mass spectrometry: 12 months' experience in

19 Gregersen N, Blakemore AI, Winter V, et al. Specific diagnosis of medium-chain acyl-CoA dehydrogenase (MCAD) deficiency in dried blood spots by a polymerase chain reaction (PCR) assay detecting a point-mutation (G985) in the MCAD gene. Clin Chim Acta 1991;203:2334.

20 Olpin SE, Manning NJ, Pollitt RJ, et al. The use of $\left[9,10-{ }^{3} \mathrm{H}\right]$ myristate, $\left[9,10-{ }^{3} \mathrm{H}\right]$ palmitate and $[9,10-$ $\left.{ }^{3} \mathrm{H}\right]$ oleate for the detection and diagnosis of medium and long-chain fatty acid oxidation disorders in intact cultured fibroblasts. Adv Exp Med Biol 1999;466:321-5.

21 Sim KG, Wiley V, Carpenter K, et al. Carnitine palmitoyltransferase I deficiency in a neonate identified by dried blood spot free carnitine and acylcarnitine profile. $\mathcal{F}$ Inherit Metab Dis 2001;24:51-9.

22 Christodoulou J, Hoare J, Hammond J, et al. Neonatal onset of medium-chain acyl-coenzyme A dehydrogenase deficiency with confusing biochemical features. 7 Pediatr ciency with conf
$1995 ; \mathbf{1 2 6}: 65-8$

23 Coates PM, Tanaka K. Workshop on molecular aspects of MCAD deficiency. Mutations causing medium-chain acyl-CoA dehydrogenase deficiency: a collaborative compilation of the data from 172 patients. In: Coates PM, Tanaka K, eds. New developments in fatty acid oxidation. New York: Wiley-Liss, 1992;499-506.

24 Heath, D. Neonatal hypoglycaemia and heterozygosity for the $985 \mathrm{~A}$ to $\mathrm{G}$ common mutation of medium chain
acyl-CoA dehydrogenase (MCAD) deficiency. Thesis, University of Western Sydney, 1996.

25 Matsubara Y, Narisawa K, Tada K. Medium-chain acylCoA dehydrogenase deficiency: molecular aspects. [Review] Eur F Pediatr 1992;151:154-9.

26 Andresen B, Dobrowolski SF, O'Reilly L, et al. The mutational spectrum in the MCAD gene of newborns identified by prospective tandem MS screening for "diagnostic" acyl-carnitnes in blood spots differs from that observed in clinically affected patients. F Inherit Metab Dis 2000;23:12.

27 Marsden D, Zykovicz T, Larson C, et al. Prevalence of fatty acid oxidation disorders and organic acidaemias in New England newborns screened by tandem mass spectrometry. F Inherit Metab Dis 2000;23:15.

28 Carpenter K, Wiley V, Sim KG, et al. Newborn screening for MCAD deficiency: the New South Wales experience. $\mathcal{F}$ Inherit Metab Dis 2000;23:16.

29 Andresen BS, Bross P, Udvari S, et al. The molecular basis of medium-chain acyl-CoA dehydrogenase (MCAD) deficiency in compound heterozygous patients: is there correlation between genotype and phenotype? Hum Mol Genet 1997;6:695-707.

30 Clayton PT, Doig M, Ghafari S, et al. Screening for medium chain acyl-CoA dehydrogenase deficiency using electrospray ionisation tandem mass spectrometry. Arch Dis Child 1998;79:109-15.

\section{Archives of Disease in Childhood}

September 2001 issue

The following articles-being published in the September 2001 issue of Archives of Disease in Childhood - may be of interest to readers of the Fetal and Neonatal edition.

Breast feeding and cognitive development at age 1 and 5 years

N K Angelsen, T Vik, G Jacobsen, L S Bakketeig

Birth weight and cognitive function at age 11 years: the Scottish Mental Survey 1932

S D Shenkin, J M Starr, A Pattie, M A Rush, L J Whalley, I J Deary

Long term complications of inferiour vena cava thrombosis

M Häusler, D Hübner, T Delhaas, E G Mühler 